\title{
Macrohabitats da Estação Ecológica de Taiamã, no contexto da Área Úmida Pantanal mato-grossense, Brasil
}

\author{
Macro-habitats of the Taiamã Ecological Station, in the context of the \\ Pantanal Wetland, Brazil
}

\author{
Angélica Vilas Boas da Frotal, Solange Kimie Ikeda-Castrillon', Daniel Luis Zanella Kantek", Carolina Joana da Silva \\ Universidade do Estado de Mato Grosso. Cáceres, Mato Grosso, Brasil \\ "Instituto Chico Mendes de Conservação da Biodiversidade/Estação Ecológica de Taiamã. Cáceres, Mato Grosso, Brasil
}

\begin{abstract}
Resumo: AÁrea Úmida Pantanal mato-grossense apresenta uma complexidade geomorfológica, hidrológica e biológica, que resulta na sua heterogeneidade ambiental. $O$ conhecimento dos macrohabitats encontrados em áreas protegidas do Pantanal pode dar suporte considerável na tomada de decisão para a sua conservação. O objetivo desta pesquisa foi identificar e caracterizar unidades funcionais, subclasses e macrohabitats da Estação Ecológica de Taiamã (EET), para contribuir com a gestão e as estratégias de manejo desta Unidade de Conservação (UC). A identificação dos macrohabitats no campo foi realizada entre os anos 2015 e 2016. Com o uso de uma cena Landsat 8, os dados foram processados por meio de classificação supervisionada. As unidades funcionais encontradas foram Áreas Permanentemente Aquáticas, Áreas de Transição Aquática e Terrestre com predominância Terrestre e Áreas Pantanosas. Seis subclasses e seis macrohabitats foram identificados. Os macrohabitats mais representativos foram Campo Flutuante (Batumes), correspondendo a 48\%, seguido de Campo Inundado, com 23\%, e de Floresta Monoespećífica de Erythrina fusca Lour. (Abobral), com 16\%. Os macrohabitats registrados na EET reproduzem o mosaico paisagístico do Pantanal, cujas funções ecológicas precisam ser protegidas por uma legislação específica, que supra as lacunas referentes à preservação das áreas úmidas brasileiras.
\end{abstract}

Palavras-chave: Planície de inundação. Diversidade de habitats. Geotecnologias. Unidades de Conservação. Conservação biológica.

Abstract: The Pantanal wetland in Mato Grosso presents a geomorphological, hydrological, and biological complexity that results in its environmental heterogeneity. Knowledge of the macrohabitats found in protected areas of the Pantanal can give considerable support to decision making for their conservation. The objective of this research was to identify and characterize functional units, subclasses, and macrohabitats of the Taiamã Ecological Station (TES), and to contribute to the management strategies of this conservation unit. The identification of macrohabitats in the field was carried out between 2015 and 2016, with the use of Landsat scene 8. The data were processed through Supervised Classification. The functional units found were Permanently Aquatic Areas, Aquatic and Terrestrial Transition Zone, and Marsh areas. Six subclasses and six macrohabitats were identified. The most representative macrohabitats were Floating Field (Batumes), corresponding to 48\%, followed by Flooded Field with 23\%, and Monospecific Forest of Erythrina fusca Lour. (Abobral) with 16\%. Registered macrohabitats in the TES reproduce the Pantanal's landscape mosaic, whose ecological functions need to be protected by specific legislation that addresses gaps in the preservation of Brazilian wetlands.

Keywords: Flood plain. Diversity of habitats. Geotechnology. Conservation Unit. Biological conservation.

FROTA, A. V. B., S. K. IKEDA-CASTRILLON, D. L. Z. KANTEK \& C. J. SILVA, 2017. Macrohabitats da Estação Ecológica de Taiamã, no contexto da Área Úmida Pantanal mato-grossense, Brasil. Boletim do Museu Paraense Emílio Goeldi. Ciências Naturais 12(2): 239-254. Autora para correspondência: Angélica Vilas Boas da Frota. Universidade do Estado de Mato Grosso. Centro de Pesquisa em Limnologia, Ecologia e Etnobiologia do Pantanal. Avenida Santos Dumont. Cáceres, MT, Brasil. CEP 78200-000 (angelicafrotaa@gmail.com).

Recebido em 25/04/2017

Aprovado em 26/10/2017

Responsabilidade editorial: Fernando da Silva Carvalho Filho

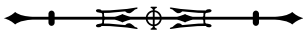




\section{INTRODUÇÃO}

A Área Úmida Pantanal mato-grossense possui uma paisagem heterogênea, com inundações periódicas que modificam suas condições ambientais. As complexidades geomorfológica, hidrológica e evolutiva da região são fatores que contribuem para a alta diversidade de habitat (Junk \& Da Silva, 1996; Harris et al., 2005), e a sua classificação é importante para discriminar as unidades de paisagem que a compõem, de modo a promover a conservação desses ambientes (Nunes da Cunha \& Junk, 2014).

O Pantanal apresenta um mosaico de paisagens e de espaços geoecológicos (Ab'Saber, 2006) que compõe muitas formações não individualizadas para os mapeamentos regionais. Há necessidade de detalhamento das áreas úmidas com diferentes resoluções para obter maiores mecanismos e compreender melhor a diversidade existente em sua paisagem (J. S. V. Silva et al., 2006), a qual se modifica constantemente, devido possuir características de flutuação do nível d'água (Da Silva \& Esteves, 1995).

Ravaglia et al. (2011) afirmam que esta complexidade de ambientes na região dificulta a definição de estratégias de manejo e de políticas públicas, sendo necessário maior detalhamento na caracterização das áreas úmidas por meio de mapas. $\bigcirc$ uso de geotecnologias e de suas diversas técnicas permite a identificação e o mapeamento de locais muitas vezes de difícil acesso, com a detecção de diferentes classes em paisagem de mosaicos, como os das áreas úmidas (Wiens et al., 1993; Silva \& Abdon, 1998; Ozesmi \& Bauer, 2002; Adam et al., 2010).

No Pantanal, diferentes classificações de paisagem por meio das geotecnologias destacam a importância de análises aprofundadas e integradas dos ambientes, devido à sua diversidade (Silva \& Abdon, 1998; Nunes da Cunha et al., 2006; Neves et al., 2009; J. S. Santos et al., 2009; Fantin-Cruz et al., 2010; Pereira et al., 2012; Paiva et al., 2014). A utilização de um sistema de classificação, portanto, pode ser uma das ferramentas básicas para formular políticas de conservação das áreas úmidas brasileiras (Brasil/MMA, 2015).
A classificação e o inventário das áreas úmidas têm se expandido mundialmente desde a Convenção de Ramsar, ocorrida em 1993 (Scott \& Jones, 1995). Muitos países já utilizam seus próprios sistemas de classificação, como os Estados Unidos (Cowardin et al., 1979), o Canadá (Warner \& Rubec, 1997), a Índia (Gopal \& Sah, 1995), a África do Sul (Ollis et al., 2015), a Argentina (Neiff, 2001) e a Bolívia (Navarro \& Maldonado, 2002). Com o uso do sistema adaptado à particularidade de cada local, os países obtiveram uma base científica para discussões em prol da conservação de seus ecossistemas.

A proposta do Sistema de Classificação e Delineamento das Áreas Úmidas Brasileiras (Brasil/MMA, 2015) estabelece um nível de identificação hierárquica com uso de classes (por exemplo, Área Úmida Pantanal mato-grossense), unidades funcionais, subclasses e macrohabitats, para identificação dessas regiões. A unidade funcional é definida como uma macrorregião com condições hidrológicas similares para as áreas úmidas, sendo descritos em outros níveis os fatores biológicos (Nunes da Cunha \& Junk, 2014).

Os macrohabitats da classificação de Áreas Úmidas (AU) foram definidos por unidades paisagísticas com uso de alguns nomes locais, pois facilitam o entendimento e a aceitação de regulamentos fundamentais para a proteção e a gestão dessas AU (Junk et al., 2014). É importante que seja estabelecido um marco regulatório para assegurar a gestão de AU brasileiras, considerando os aspectos de variedade de ecossistemas em uma classificação (Irigaray, 2015).

A caracterização de áreas úmidas no contexto de um sistema de classificação pode gerar novos conhecimentos e informações que forneçam subsídios aos órgãos ambientais e tomadores de decisão. Assim, estudos detalhados em Unidades de Conservação inseridas no ecossistema de Área Úmida tornam-se uma importante estratégia quanto à gestão, ao manejo e à conservação desses ecossistemas. Esta pesquisa teve como objetivo identificar e caracterizar unidades funcionais, subclasses e macrohabitats da Estação 
Ecológica de Taiamã, na Área Úmida Pantanal matogrossense, Brasil.

\section{MATERIAL E MÉTODOS}

\section{ÁREA DE ESTUDO}

A Estação Ecológica de Taiamã (EET) é uma Unidade de Conservação (UC) de proteção integral (Brasil, 2000), localizada entre os meridianos W $57^{\circ} 24^{\prime}$ e W $45^{\circ} 40^{\prime}$ e paralelos $\mathrm{S} 16^{\circ} 48^{\prime}$ e $\mathrm{S} 16^{\circ} 58^{\prime}$, no município de Cáceres, bioma Pantanal (Figura 1). Encontra-se na bacia do alto rio Paraguai (ANA, s. d.) e ocupa uma área de 11.555 ha do Pantanal mato-grossense, entre os rios Paraguai e Bracinho. Possui, em seu entorno, uma zona de amortecimento ('campo') protegida por uma Instrução Normativa (09/2009) e uma Reserva Particular do Patrimônio Natural (RPPN) (Brasil/MMA, 2017).

De acordo com a classificação de Koppen (1948), o clima da região é do tipo Aw (quente e úmido), com precipitação anual de 1.500 mm, com maior intensidade nos meses de janeiro, fevereiro e março. As temperaturas médias anuais giram em torno de 20 a $32^{\circ} \mathrm{C}$, com alternância nos períodos de cheia e de estiagem.

A EET localiza-se na unidade geomorfológica caracterizada por deposição recente de sedimentos da Formação Pantanal, com solos hidromórficos de

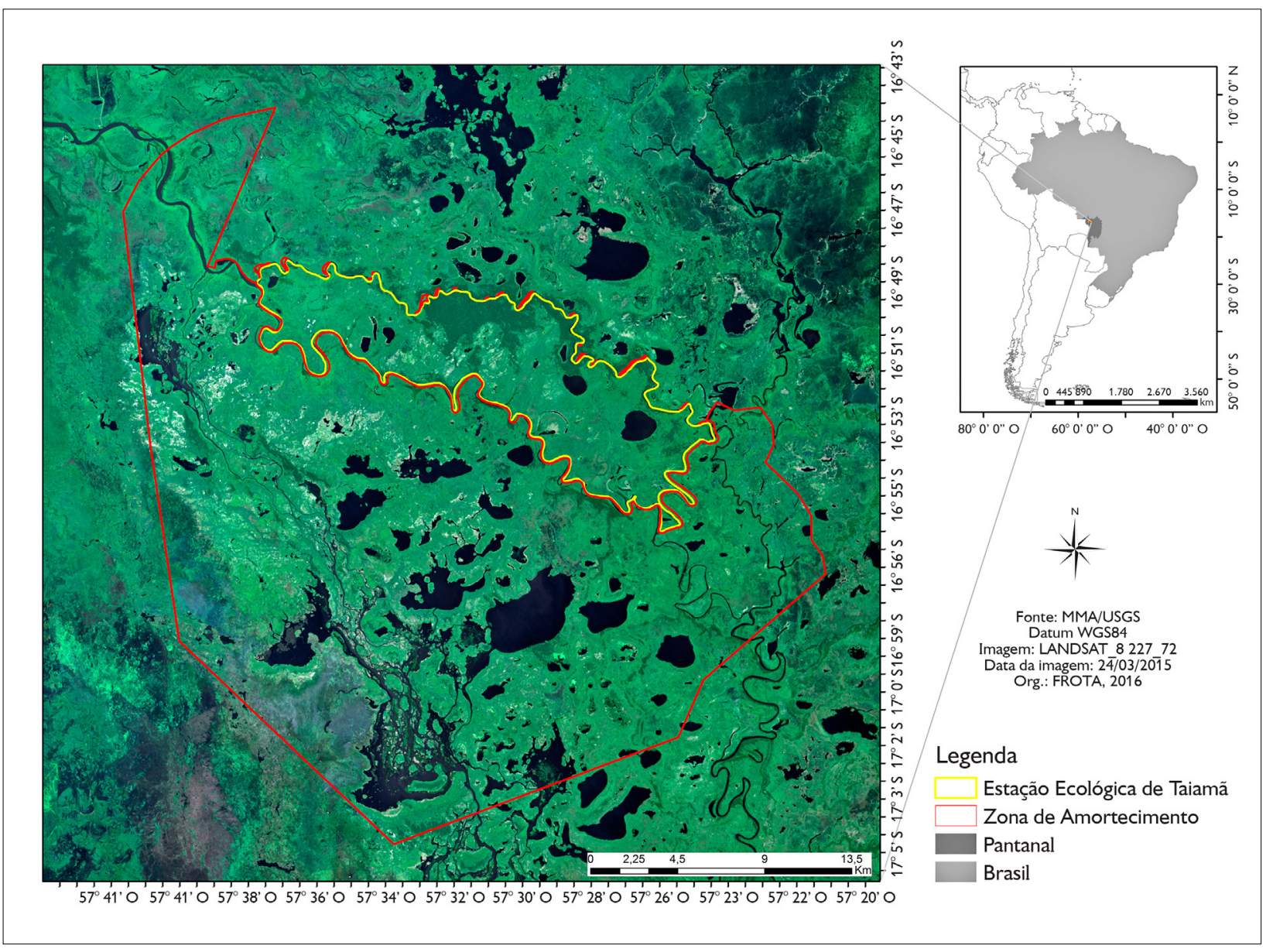

Figura 1. Localização e delimitação da área de estudo: Estação Ecológica de Taiamã, representada em uma ilha fluvial, entre os rios Paraguai e Bracinho, Pantanal mato-grossense, Brasil. Fonte: adaptado de Brasil/MMA (s. d.).

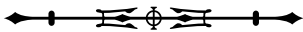


forte tendência a inundações periódicas e prolongadas, originárias do processo de sedimentação (Franco \& Pinheiro, 1982; Souza \& Cunha, 2007). Veloso et al. (1991) classificam a vegetação desta área como Floresta Estacional Semidecidual Aluvial, formação encontrada na depressão pantaneira margeada pelo rio Paraguai.

\section{METODOLOGIA}

A identificação e a caracterização das unidades funcionais, das subclasses e dos macrohabitats de Área Úmida na EET foram baseadas em um Sistema de Classificação de Áreas Úmidas (Brasil/MMA, 2015). Informações climáticas, hidrológicas e espaciais foram coletadas, sendo realizadas visitas na área entre os anos de 2015 e 2016 (licença obtida no Sistema de Autorização e Informação em Biodiversidade - SISBIO n. 50928-1). O processamento dos dados foi feito por meio de um Sistema de Informação Geográfica (SIG) de forma integrada (Rosa, 2005).

\section{Coleta de dados}

Dados sobre fatores climáticos e hidrológicos, como a temperatura, a umidade, a precipitação da chuva, o nível d'água e a vazão do rio Paraguai, correspondentes aos anos de 2015 e 2016, foram adquiridos na Agência Nacional de Águas (ANA) e no Instituto Nacional de Meteorologia (INMET). Os dados vetoriais foram adquiridos no Ministério do Meio Ambiente (MMA) e na Secretaria de Estado de Planejamento e Coordenação (SEPLAN). Os dados matriciais foram coletados por meio de imagens Landsat-8 sensor Operacional Terra Imager (OLI), órbita e ponto 227/72, com data de 31/08/2015 (USGS, s. d.). Os pontos de controle foram feitos a partir de observações dos macrohabitats e da descrição das principais características verificadas. As visitas foram realizadas no período de cheia e de estiagem, com marcação dos pontos e registro fotográfico.

\section{Processamento dos dados}

O sistema de projeção adotado foi o Universal Transversal de Mercator (UTM), reprojetado na Zona 21S, Datum de referência WGS 84, com escala de 1:80.000. 0 pré-processamento foi feito com a composição R6G5B4, para trabalhar nas bandas multiespectrais ( 1 a 7 e 9 para L8), com resolução espacial de $30 \mathrm{~m}$, de modo a detalhar ainda mais os alvos a serem caracterizados, como floresta inundável, corpos d'água e campos inundados.

O método para elaboração do mapa temático foi o de classificação digital de imagens de satélite (Lillessand \& Kiefer, 1999), por meio da classificação supervisionada (Mendes, 1987). Os pontos de uma imagem foram associados a classes ou a categorias pré-definidas, para a elaboração das amostras de treinamento e para gerar o arquivo de assinatura (.gsg). Após o processamento, foi realizada uma caracterização estatística das reflectâncias (resposta espectral) para cada classe (Rosa, 2009). Nesse estudo, estas classes foram denominadas a partir de seus níveis hierárquicos da classificação de áreas úmidas, iniciando-se com as categorias de unidades funcionais, sendo posteriormente identificadas as categorias de subclasses e de macrohabitats.

\section{Análise dos dados}

Uma tabela de atributos das camadas foi gerada a partir dos dados processados, com a representação da classe e do tamanho da área. As áreas dos canais do rio foram excluídas nas análises de tamanho dos macrohabitats, por não representarem toda a sua extensão no recorte do Shapefile utilizado. A validação das classes amostrais foi feita a partir de uma matriz de confusão, representando a distribuição de pixels (\%) de forma correta ou erroneamente, em um conjunto de dados da classificação e da verdade terrestre para acurácia (Colgaton, 1991). As amostras de treinamento foram utilizadas, configurando-se como a representação da assinatura espectral das classes (INPE, 2006).

O Índice de Kappa (IK) foi utilizado para expor a confiabilidade e a qualidade das informações, com um coeficiente de concordância que varia de 0 a 1, sendo que, quanto mais próximo de 1 , maior é a qualidade da 
classificação (Cohen, 1960). Com o cálculo realizado, foi possível obter o IK, o índice de concordância e a exatidão global. $\bigcirc$ método de classificação aplicado mostrou-se satisfatório, com IK e exatidão global igual a 0,97 e com índice de concordância com o valor de 68,51. Segundo a escala de importância proposta por Landis \& Koch (1977), os valores entre 0,80 e 1,00 são considerados excelentes.

\section{RESULTADOS E DISCUSSÃO}

\section{FATORES CLIMÁTICOS E HIDROLÓGICOS}

No ciclo hidrológico de 2015-2016, os fatores climáticos avaliados mensalmente de temperatura máxima e mínima $\left({ }^{\circ} \mathrm{C}\right.$ ) estiveram entre 37 e $21{ }^{\circ} \mathrm{C}$, com umidade relativa do ar (\%) atingindo de $82 \%$ a $32 \%$. Pode-se observar que, na estação seca, houve baixa umidade relativa do ar, acompanhada de temperaturas mais altas, principalmente nos meses de agosto, setembro e outubro de 2015 (Figura 2).

A precipitação mais elevada no ano de 2015 foi no mês de fevereiro (218,4 mm) e, para o ano de 2016, foi durante o mês de janeiro $(285,6$ mm). O nível d'água do rio Paraguai acompanhou a precipitação, e alcançou o pico mais alto em março de $2015(447,70 \mathrm{~cm})$ e de $2016(407,39 \mathrm{~cm})$. Os períodos com menor precipitação foram em agosto de 2015 (1,4 mm) e julho de 2016 (0). Em relação ao nível d'água, os menores foram em setembro de 2015 (98,91 cm) e outubro de 2016 (95,59 $\mathrm{cm})$, conforme a Figura 3.

A maior vazão no ano de 2015 foi durante o mês de março $\left(1.098,92 \mathrm{~m}^{3} / \mathrm{s}\right)$. Já para o ano de 2016, a maior vazão ocorreu no mês de fevereiro $\left(880,15 \mathrm{~m}^{3} / \mathrm{s}\right)$. Observa-se que este aspecto esteve diretamente relacionado com o nível de precipitação, sendo maior durante os meses de cheia, e menor durante os meses de estiagem, conforme a Figura 4.

Da Silva \& Girard (2004) afirmam que essa região costuma apresentar clima quente, com estação

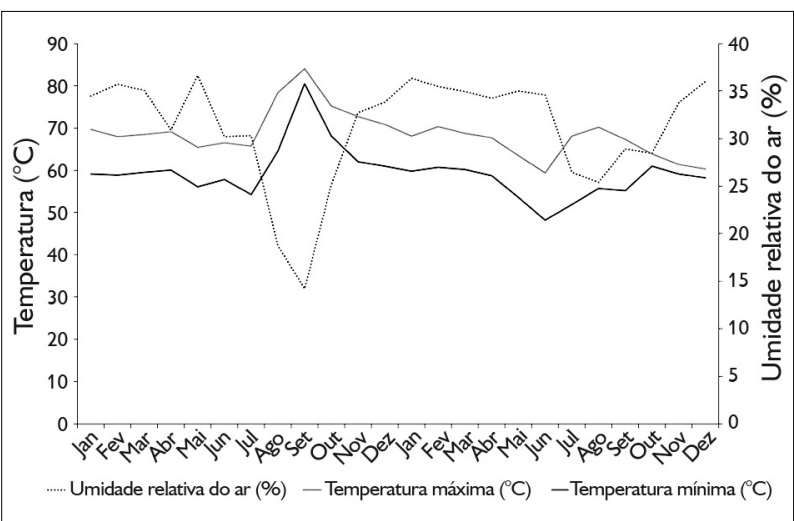

Figura 2. Temperatura do $\operatorname{ar}\left({ }^{\circ} \mathrm{C}\right)$ e umidade relativa do ar (\%) durante o ciclo hidrológico de 2015-2016 na Estação Meteorológica Automática do município de Cáceres, Mato Grosso. Fonte: adaptado de INMET (s. d.).

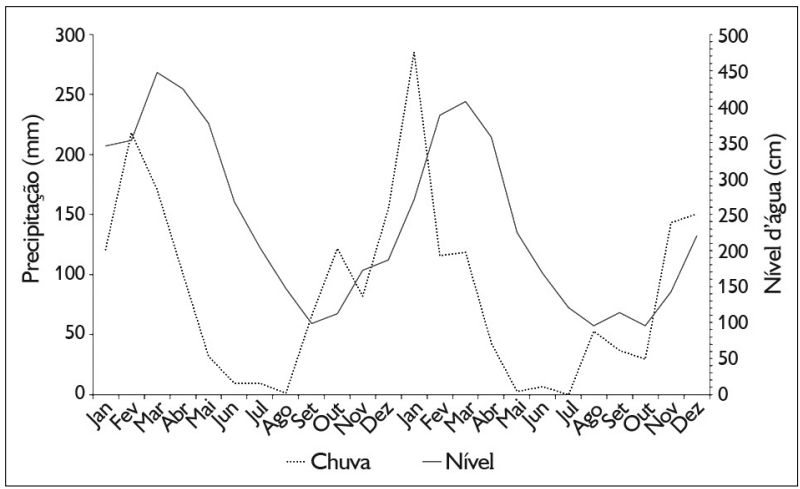

Figura 3. Precipitação $(\mathrm{mm})$ e nível d'água $(\mathrm{cm})$ do rio Paraguai durante o ciclo hidrológico de 2015-2016 na Estação Hidrológica do município de Cáceres, Mato Grosso. Fonte: adaptado de ANA (s. d.).

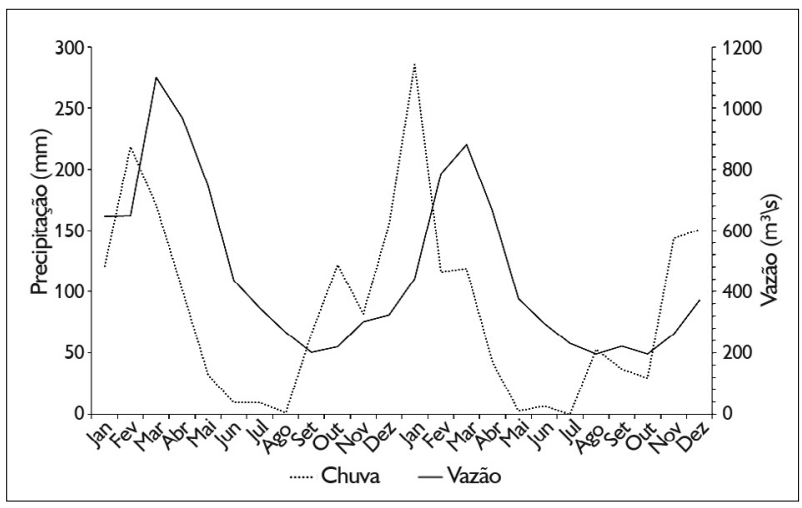

Figura 4. Precipitação $(\mathrm{mm})$ e vazão $\left(\mathrm{m}^{3} / \mathrm{s}\right)$ do rio Paraguai no município de Cáceres, Mato Grosso, durante o ciclo hidrológico de 2015-2016 na Estação Hidrológica do município de Cáceres, Mato Grosso. Fonte: adaptado de ANA (s. d.). 
seca entre os meses de maio a setembro, e estação chuvosa no período de outubro a abril. No rio Paraguai, em Cáceres, o pico de cheia ocorre geralmente em fevereiro e março, assim como o pico da precipitação (Da Silva \& Girard, 2004). Nota-se que em janeiro de 2016 também houve um pico de precipitação acompanhado de alto nível d'água.

No ciclo hidrológico de 2015 e 2016, observou-se que a variabilidade da precipitação em Cáceres esteve dentro de médias anuais já estudadas. Porém, a mínima encontrada neste ciclo esteve acima das mínimas registradas em séries anteriores. Em análise histórica dos dados pluviométricos de Cáceres, Pizzato et al. (2012) encontraram grande variabilidade na precipitação anual, com mínima de 972,9 mm no ano de 1985, e máxima de $1.624,1 \mathrm{~mm}$ no ano de 1998. De acordo com Santos et al. (2012), essa variabilidade de chuva na região de Cáceres pode atingir de $950 \mathrm{~mm}$ a 1,800 mm anuais.

Com as modificações do ciclo hidrológico anual na região de Cáceres, mudanças na cobertura vegetal também ocorrem devido ao processo de inundação. Segundo Ikeda-Castrillon et al. (2011b), as ilhas nesta região são submetidas à inundação anual por um período de aproximadamente seis meses, geralmente de novembro a abril, entre o início da cheia e o fim da enchente. Nesse sentido, o aumento ou a diminuição do nível d'água do rio que se expande para as margens na planície influencia diretamente as características dos macrohabitats que passam por essas mudanças anuais.

\section{MACROHABITATS IDENTIFICADOS}

Três unidades funcionais foram identificadas na EET (Áreas Permanentemente Aquáticas, Áreas de Transição Aquática e Terrestre (ATTZ) com predominância Terrestre e Áreas Pantanosas), de seis descritas para a Área Úmida Pantanal mato-grossense (Brasil/MMA, 2015). Seis subclasses (Floresta Monoespecífica, Floresta Poliespecífica, Campo Limpo Natural, Canais de Rios, Lagos e Pântanos de Plantas Herbáceas) foram encontradas na área, das 16 classificadas para a região (Brasil/MMA, 2015).

A Área Úmida Pantanal mato-grossense tem o registro de 56 macrohabitats (Brasil/MMA, 2015). Destes, seis foram identificados na EET (Floresta com Monodominância de Erythrina fusca (Abobral), Arbustais e Florestas Pioneiras ao Longo de Rios, Campo Inundado, Partes Centrais dos Canais, Lagos de Depressão e Pântanos Flutuantes - Batumes) no período de estiagem (Tabela 1).

As categorias de unidades funcionais dominantes na paisagem avaliada foram: Áreas Pantanosas (48\%), Áreas de Transição Aquática e Terrestre (ATTZ) com predominância Terrestre (47\%) e Áreas Permanentemente Aquáticas (5\%). Cada uma das subclasses encontradas na EET foi representada por um tipo de macrohabitat observado em campo (Figura 5).

Tabela 1. Unidades funcionais, subclasses e macrohabitats encontrados na Estação Ecológica de Taiamã, Área Úmida Pantanal mato-grossense, identificados no Sistema de Classificação de Áreas Úmidas Brasileiras (Brasil/MMA, 2015).

\begin{tabular}{c|c|c}
\hline \multicolumn{2}{c}{ Classe Pantanal mato-grossense, Estação Ecológica de Taiamã, Mato Grosso } \\
\hline Unidade funcional & Subclasse & Macrohabitat \\
\hline \multirow{2}{*}{$\begin{array}{c}\text { Área de Transição Aquática e Terrestre } \\
\text { (ATTZ) com predominância Terrestre }\end{array}$} & Floresta Monoespecífica & $\begin{array}{c}\text { Floresta com Monodominância de } \\
\text { Erythrina fusca (Abobral) }\end{array}$ \\
\cline { 2 - 3 } & Floresta Poliespecífica & Arbustais e Florestas Pioneiras ao Longo de Rios \\
\cline { 2 - 3 } & Campo Limpo Natural & Campo Inundado \\
\hline \multirow{2}{*}{ Área Permanentemente Aquática } & Canais de Rios & Partes Centrais dos Canais \\
\cline { 2 - 3 } & Lagos & Lagos de Depressão \\
\hline Áreas Pantanosas & Pântanos de Plantas Herbáceas & Pântanos Flutuantes (Batumes)
\end{tabular}

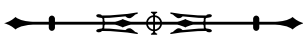



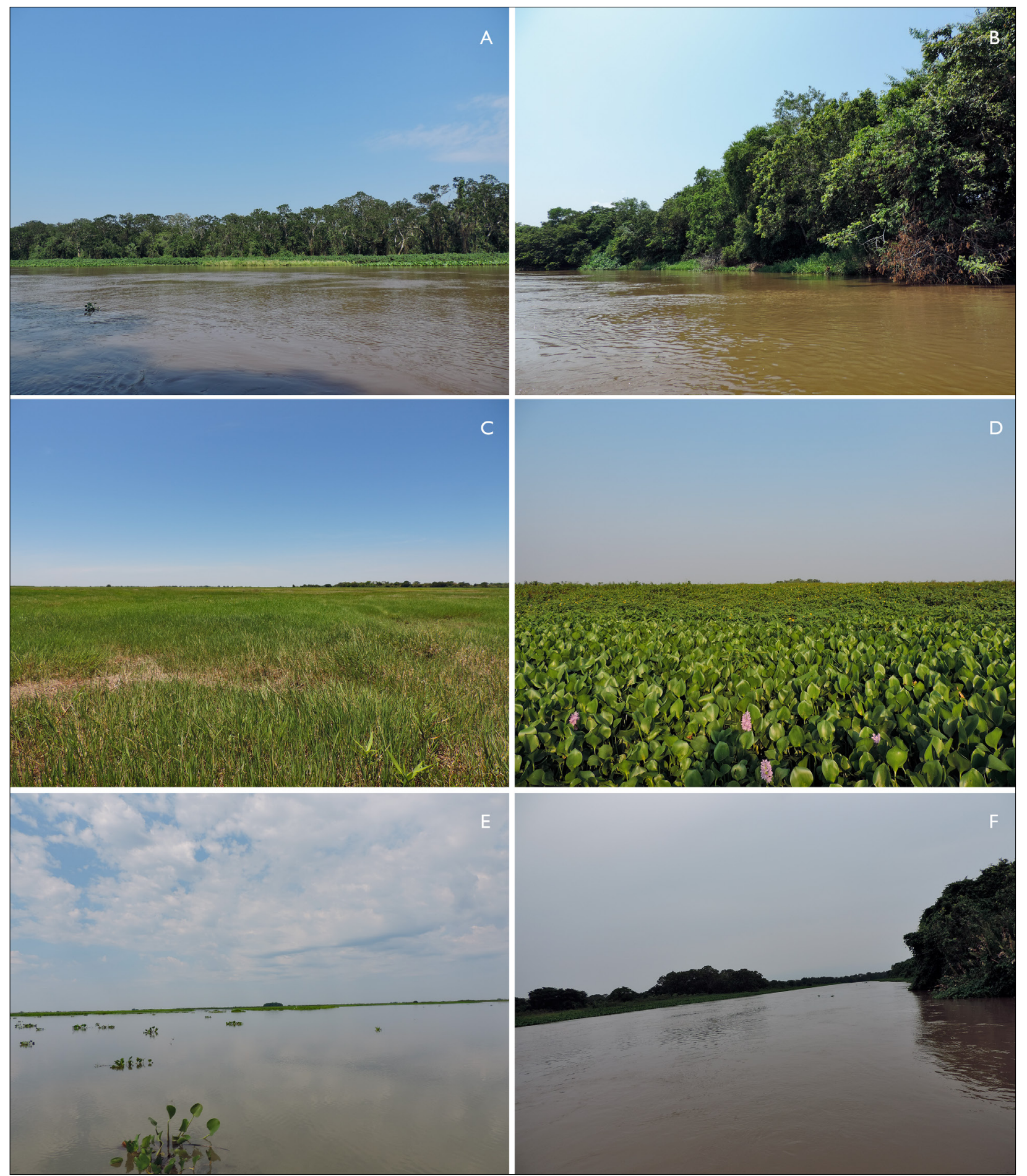

Figura 5. Categorias de macrohabitats da Estação Ecológica de Taiamã, Área Úmida Pantanal mato-grossense, Brasil: A) Floresta Monodominante de Abobral; B) Arbustais e Florestas Pioneiras; C) Pântanos Flutuantes (Batumes); D) Campo Inundado; E) Lagos; F) Canal do Rio. Fotos: Angélica Vilas Boas da Frota (2015).

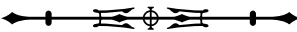


As ATTZ são muito comuns na Área Úmida do Pantanal. Apenas 20 a $30 \%$ são de habitats cobertos permanentemente por água ou encharcados, e todo o restante é composto por zonas de transição (Nunes da Cunha \& Junk, 2009). Na EET, há maior dominância de áreas de campo e habitats mais encharcados. Isso se associa a uma diminuição drástica no gradiente topográfico desse segmento do rio Paraguai, conforme A. Silva et al. (2006), tornando, assim, a área da EET um local particular e muito dinâmico quanto aos processos de inundação.

A dinâmica de inundação e a presença de macrófitas aquáticas foram observadas na EET. Isso indica a possibilidade de ocorrer o macrohabitat de áreas sazonalmente cobertas com macrófitas aquáticas flutuantes e enraizadas emersas. Porém, houve dificuldade em distinguir a classe na cena L8, devido à sua cobertura apresentar semelhança com as herbáceas. Dessa forma, estudos de campo mais refinados a respeito do grupo de macrófitas aquáticas podem facilitar a distinção entre os macrohabitats.

A unidade funcional Áreas Pantanosas recobriu a maior parte da área de estudo. Os pântanos são locais inundados ou encharcados em todos os períodos hidrológicos, representando 7,4\% da vegetação do Pantanal (Silva et al., 2000). Esse tipo de unidade funcional possui funções ecológicas de filtragem de água e também servem como refúgio para animais (Pott et al., 2001). Destaca-se a importância desses pântanos, pois são os ambientes mais afetados por alterações hidrológicas ou antrópicas (Nunes da Cunha et al., 2014).

As ATTZ com macrohabitats de florestas identificadas na EET estiveram localizadas principalmente nas margens dos rios, com a formação monodominante estendendo-se até o interior da ilha. De acordo com Silva et al. (2000), a mata ciliar está presente ao longo do rio Paraguai, totalizando 2,4\% da vegetação do Pantanal. Os macrohabitats da EET, assim como boa parte das áreas do Pantanal, apresentam, segundo Ab'Saber (2006), um mosaico de paisagens conectadas e ampla diversidade de ambientes.
Com a identificação dessas categorias em campo, a coleta de pontos de controle e por meio de observação da cena, atribuíram-se diferentes classes de macrohabitats para o uso da técnica de classificação supervisionada. A partir dela, foi possível gerar um mapa temático para representar cada macrohabitat da EET (Figura 6).

Esse sistema de classificação utilizado baseia-se em aspectos estruturais e funcionais das planícies inundáveis, discutidos por Wantzen et al. (2005). Segundo os autores, esses aspectos dependem das interações dinâmicas dos processos hidrogeomorfológicos e biológicos da região, que apresenta setores diferenciados no rio Paraguai.

Trabalhos de identificação dos tipos de ambientes na Área Úmida Pantanal utilizam métodos e escalas diferenciados, que obtêm diferentes resultados. Os dados sobre a vegetação e a inundação são os principais parâmetros utilizados para discriminar esses elementos, existindo atualmente diversas técnicas para compreender esses mosaicos.

Por meio de imagens orbitais e fotografias aéreas, Ponzoni \& Hernandez-Filho (1989) realizaram uma caracterização da fisionomia vegetal no Parque Nacional do Pantanal mato-grossense (PARNA Pantanal mato-grossense). As principais classes que os autores encontraram foi água, campo inundado, campo úmido, campo, vegetação arbórea-arbustiva, arbórea baixa e arbórea, com variações nos tamanhos entre cada período. Houve maior distinção dos ambientes durante a estiagem. É possível observar que há semelhança quanto aos tipos de classes encontradas no PARNA em relação às classes da EET.

No Pantanal do município de Cáceres, em Mato Grosso, a identificação foi realizada por meio do uso da geotecnologia Land System. As unidades ambientais encontradas foram 16 áreas não inundáveis, nove inundáveis e oito inundadas (Neves et al., 2009). S. A. Santos et al. (2009), a partir de um levantamento aéreo no Pantanal de Poconé, encontraram que a maior parte da unidade de paisagem na área são as florestas sazonalmente inundáveis, dominadas pelo cambará (Vochysia divergens Pohl),

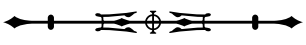




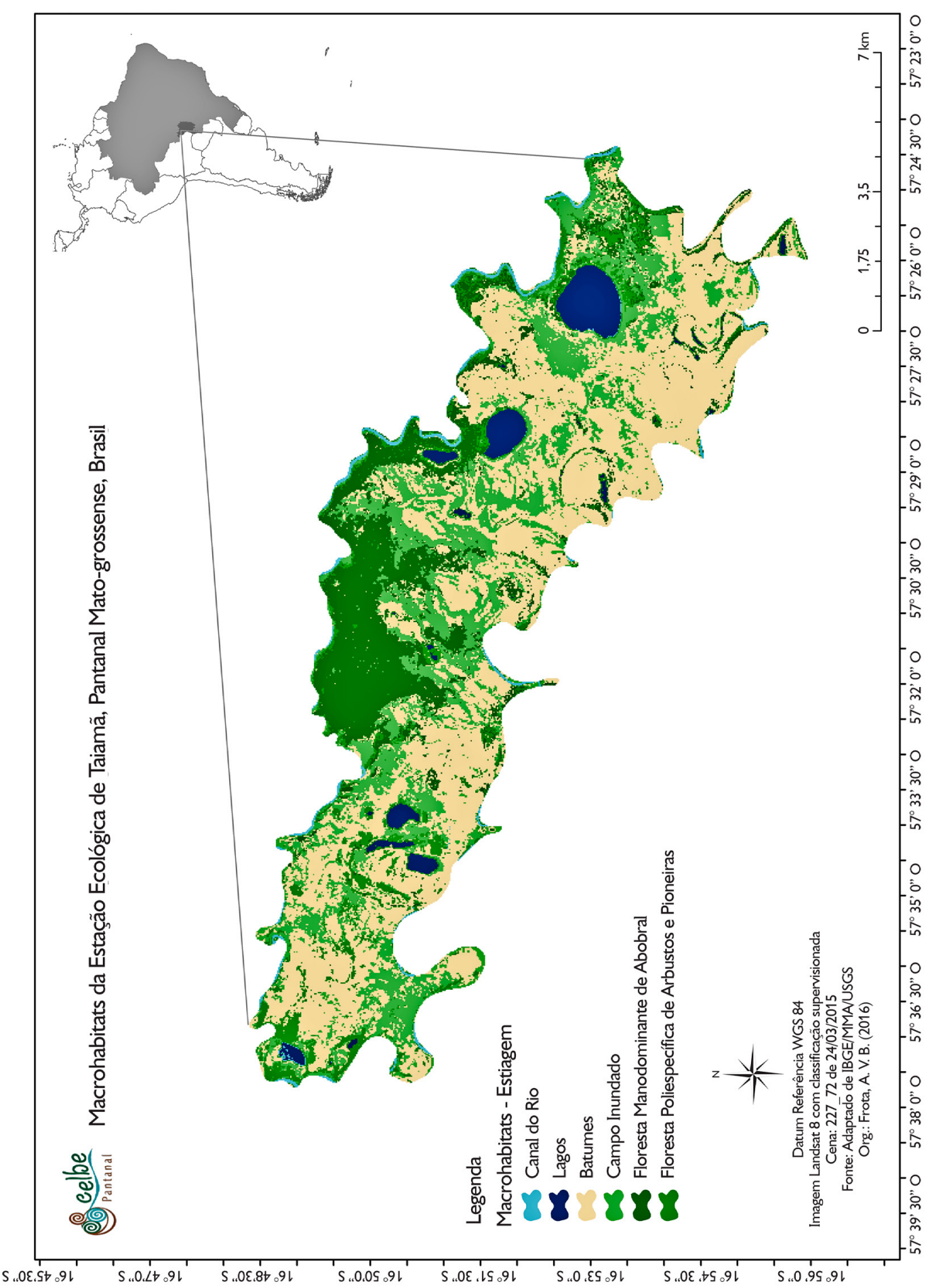

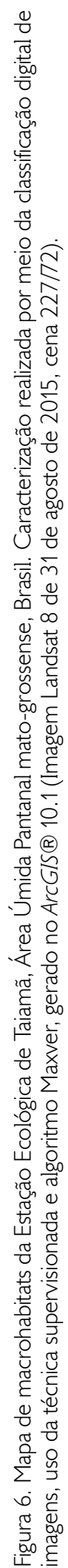

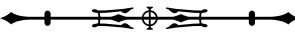


seguidas das áreas savânicas sazonalmente inundáveis. As formações florestais no Pantanal estão sujeitas a uma intensa dinâmica populacional, associada ao processo de espalhamento/retração de monodominâncias (Arieira \& Nunes da Cunha, 2012), o que foi possível também de ser observado na EET, em razão da presença de monodominância da Erythrina fusca.

A avaliação da vegetação e do grau de inundação em duas áreas no Pantanal foi realizada por Ravaglia et al. (2011), que puderam identificar quatro classes e dez unidades de paisagem. Em um contexto de bioma, Pereira et al. (2012) avaliaram, com análise multitemporal, os principais elementos da paisagem pantaneira, descrevendo 16 unidades de paisagem nesta área úmida. Desse modo, os diversos métodos apresentados demonstram aprimoramento das técnicas ao longo do tempo para identificar os ambientes no Pantanal, as quais foram adaptadas à necessidade de cada estudo e análise, sendo a escala fundamental para a escolha da técnica.

\section{TAMANHO DOS MACROHABITATS}

Em relação ao tamanho dos macrohabitats, os mais representativos nesta pesquisa foram o Campo de Batume (48\%), seguido de Campo Inundado (24\%) e de Floresta Monoespecífica de Abobral (16\%). As áreas de Floresta Poliespecífica de Arbustos e Pioneiras (8\%) e Lagos (4\%) foram menos representativas quanto ao tamanho na área delimitada (Figura 7).

$\mathrm{Na}$ estiagem, o tamanho dos macrohabitats por quilômetro quadrado $\left(\mathrm{km}^{2}\right)$ indicou que os Batumes compreendem cerca de 55.037,713 km²; os Campos Inundados, 26.857,360 km²; as Florestas Monodominantes de Erythrina fusca (Abobral), 17.990,452 km²; as Florestas Poliespecíficas Pioneiras e Arbustivas, 9.274,258 km²; e os Lagos, 4.622,625 km². Ressalta-se que o tamanho dos macrohabitats representados nessa análise compreende a dinâmica de inundação que ocorreu durante o período avaliado, podendo, assim, ter uma variação devido às alterações que acontecem no regime hidrológico.

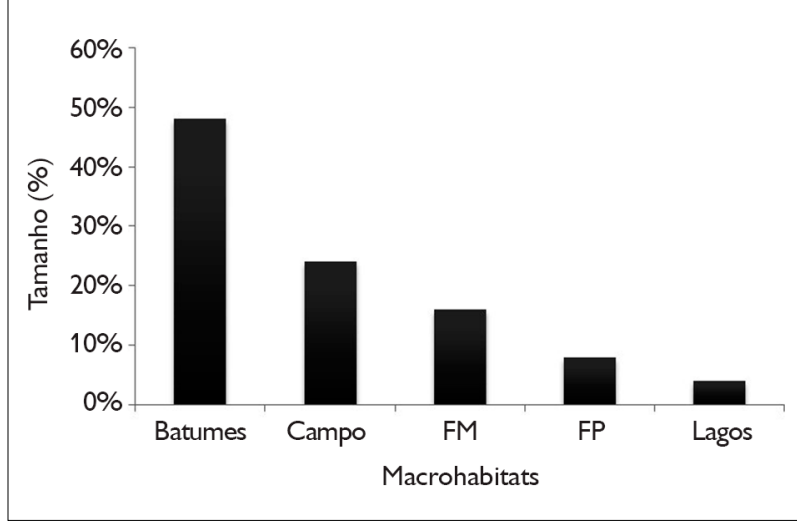

Figura 7. Percentual de macrohabitats da Estação Ecológica de Taiamã, Área Úmida Pantanal mato-grossense, Brasil. Caracterização realizada por meio da classificação digital de imagens, uso da técnica supervisionada e algoritmo Maxver, gerado no $\operatorname{ArCGIS} \AA^{10} 1$ (imagem Landsat 8 de 31 de agosto de 2015, cena 227/72). Legenda: Floresta Monoespecífica de Erythrina fusca Abobral (FM), Arbustais e Florestas Pioneiras (FP), Pântanos de Batumes, Campo Inundado, Lagos.

A diversidade morfológica dos habitats no rio Paraguai é atribuída por Wantzen et al. (2005) em virtude das mudanças na conectividade hidrológica, relacionadas a aumento das plantas aquáticas (como Nymphaea, Ludwigia, Echinodorus, Utricularia, Cabomba e várias Pontederiaceae) ou aos períodos plurianuais de cheia e de estiagem. Segundo Pott et al. (2011), a variação nos tamanhos dos macrohabitats pode, então, estar relacionada com os períodos hidrológicos, principalmente quanto ao aumento dessas plantas na época de cheia, que respondem bem à dinâmica desse período.

Ponzoni \& Hernandez-Filho (1989) afirmam que, durante o período de cheia, o número de classes pode diminuir, devido a um mascaramento da verdadeira área ocupada por essas plantas aquáticas. De acordo com Fantin-Cruz et al. (2010), os aspectos topográficos e geomorfológicos da área devem ser considerados também, visto que há diferenciação quanto à inundação nessas unidades fitofisionômicas.

\section{CARACTERIZAÇÃO DOS MACROHABITATS}

As áreas de Floresta Monoespecífica de Abobral são compostas por monodominância de Erythrina fusca Lour., 
estendendo-se nas margens dos rios Bracinho e Paraguai. Possuem uma população agrupada principalmente nas margens do rio Bracinho, que vai até o interior da EET. De acordo com Nunes da Cunha et al. (2014), as áreas com dominância de $E$. fusca podem permanecer inundadas até seis metros, permanecendo assim por até oito meses.

O termo monodominância descreve as florestas que apresentam mais de $50 \%$ do número de indivíduos da comunidade de uma única espécie (Hart et al., 1989). Segundo Connell \& Lowman (1989), também são incluídas, para caracterizar essas florestas, a área basal, a biomassa ou a cobertura do dossel. Em estudo das monodominâncias no Brasil, Nascimento et al. (2015) concluíram que um fator apto a explicar a dominância de $V$. divergens no Pantanal é o alagamento, diferenciando-a das florestas monodominantes de terra firme. Na EET, há o fator alagamento, sendo que a formação monodominante de E. fusca é única e foi recentemente estabelecida na área.

As espécies de plantas tolerantes a prolongados períodos de inundação e a solos com elevada acidez podem apresentar vantagens competitivas na ocupação destas áreas úmidas (Arieira \& Nunes da Cunha, 2012). Os períodos longos de inundação são favoráveis à germinação de E. fusca na EET (Gris et al., 2016a). O local possui grande concentração de enxofre no solo, fator que pode favorecer o desenvolvimento das plântulas durante o período da estiagem (Gris et al., 2016b).

Os Arbustais e as Florestas Pioneiras são áreas de mata ciliar, localizados na porção mais baixa do canal de rios. Estão presentes nas margens dos rios Paraguai e Bracinho. Pouco representadas na ilha (8\%), entretanto, fornecem áreas de refúgio e de alimentação para diversas espécies de aves frugívoras e insetívoras. São compostas por espécies vegetais como Bactris sp., Triplaris americana L., Sapium obovatum Klotzsch ex Mull, Spondias mombin L., Inga vera Willd., comuns nas ilhas do rio Paraguai (Ikeda-Castrillon et al., 2011b).

As áreas inundáveis sugerem baixa riqueza e diferença de densidade para a diversidade da vegetação arbórea na região do Pantanal mato-grossense, em Cáceres
(Ikeda-Castrillon et al., 2011b). No entanto, a distribuição também é influenciada pelas variáveis de atributo do solo e de diferenciação topográfica e hidrológica, que podem ser distinguidas como áreas de campo inundado e áreas com vegetação florestal (Ikeda-Castrillon et al., 2011a).

O macrohabitat de Campo Inundado é composto por áreas abundantes na ilha, formadas por plantas herbáceas, sendo que na fase aquática predominam as macrófitas aquáticas. As áreas de campo apresentam características diferenciadas, de acordo com a sazonalidade local, com predominância de plantas aquáticas como Eichhornia sp. (aguapés), sendo que, em alguns locais, há formação de praias durante o período de estiagem.

A sazonalidade do Pantanal gera uma sucessão cíclica das espécies do campo, sendo algumas comuns em ambas as fases (Rebellato \& Nunes da Cunha, 2005). O pulso de inundação no Pantanal influencia a riqueza, a diversidade, a composição e a cobertura das formas de vida dessas espécies (Rebellato et al., 2012).

As áreas de campo inundado ocupam a porção oeste do Pantanal, próxima ao rio Paraguai. As proporções entre campo seco e campo inundado alternam-se em função da precipitação local e do aporte de água por rios intermitentes ou não e também no que se refere à época do ano. A transição entre campo e cerrado é bastante dinâmica, sendo determinada pela umidade do solo (Silva et al., 2000).

As áreas de Batume foram dominantes no espaço avaliado e localizadas em toda a porção da ilha, as quais possuem dinâmica sucessional com diferentes estágios e encontram-se entre os limites dos ecossistemas aquático e terrestre. Apresentam formas biológicas epifitas e epífitas casuais, sendo as famílias mais numerosas Cyperaceae e Poaceae (Pivari et al., 2008).

Campos de batumes são formados por camadas de matéria orgânica flutuante (resto de vegetação aquática, raízes e rizomas), que acompanham a flutuação do nível d'água (Pott \& Pott, 2000). Com a retenção desses restos vegetais, ocorre aumento desta camada flutuante, que pode alcançar vários metros de profundidade (Da Silva, 1984). 
Esta camada apresenta solo orgânico que fornece maior capacidade de suporte e forma um substrato de fixação para arbustos e árvores (Da Silva, 1984), além de proporcionar local de refúgio para a fauna, como o caso de Blastocerus dichotomus (Illiger, 1815) (cervo-dopantanal), Panthera onca (Linnaeus, 1758) (onça-pintada), Hydrochoerus hydrochaeris (Linnaeus, 1766) (capivara), Caiman yacare (Daudin, 1802) (jacaré-do-pantanal) e diversas espécies de aves de áreas úmidas.

Os Lagos, denominados localmente de baías (Da Silva \& Silva, 1995), estão localizados dentro da ilha e foram observados apenas pelas imagens de satélite. Apresentam diversas formas (circulares e irregulares) e tamanhos. Esses macrohabitats são um dos mais estudados na Área Úmida Pantanal, desde seus aspectos físico-químicos até os biológicos e os etnobiológicos (Da Silva \& Esteves, 1995; Da Silva \& Figueiredo, 1999; Pinto et al., 1999; Da Silva \& Simoni, 2012; Bleich et al., 2009). Segundo Costa et al. (2015), os mecanismos responsáveis pela formação da diversidade de lagos na região do Pantanal estão provavelmente vinculados à combinação de isolamento de água subterrânea, recarga de água superficial, evaporação, descarga e processos biogeoquímicos nas bacias hidrográficas do lago.

O Canal do Rio, enquanto macrohabitat, está representado na EET pelos canais do rio Paraguai, que delimitam a EET. Segundo J. S. V. Silva et al. (2006), apresentam alta sinuosidade e passam por uma dinâmica de processos autogênicos e por variáveis externas que alteram o gradiente hidráulico. Souza et al. (2014) afirmam que as mudanças na extensão do canal do rio Paraguai estão associadas à migração lateral, que reduz a sua sinuosidade, vinculada ao rompimento do colo do meandro, com aparecimento de novos canais, originando novas baías.

No rio Paraguai, há transformações nas características limnológicas dos corpos d'água, causadas pelas interações entre os ambientes aquáticos e os terrestres, conhecidas como 'dequada' (Da Silva, 1984; Calheiros \& Ferreira, 1996). Com as primeiras chuvas e a elevação do nível d'água, aumenta a área de inundação, os processos de decomposição e de escoamento superficial, que carregam águas comprometidas para os canais de drenagem e para o leito do rio. Esse fenômeno é muito dinâmico e depende das características hidrológicas de cada ano (Calheiros \& Ferreira, 1996).

As modificações que ocorrem nos ecossistemas de áreas úmidas interiores, sujeitas a pulso de inundação, como a variação em sua duração e em sua profundidade, tornam esses ambientes muito dinâmicos e complexos. Essas alterações causam mudança na cobertura vegetal e, consequentemente, no tamanho, na forma, na presença ou na ausência de um tipo de macrohabitat de Área Úmida no Pantanal.

\section{CONCLUSÃO}

O estudo apresentou as principais tipologias de macrohabitats da Estação Ecológica de Taiamã, a partir de suas identificação e caracterização por meio do uso de geotecnologias. A compreensão da dinâmica hidrológica das áreas úmidas é de extrema importância para que se conheçam os diferentes tipos de macrohabitats, bem como o seu funcionamento. Recomenda-se a realização de estudos aprofundados sobre a comunidade vegetal para determinar outros tipos de macrohabitats existentes na unidade.

A delimitação espacial de cada tipo de macrohabitat e as suas descrições podem ser úteis em várias ações de manejo nesta Unidade de Conservação, como a elaboração de estratégias de combate a incêndios florestais, visto que diferentes ambientes respondem de forma variada a este tipo de pressão. Este estudo deverá ser muito útil também para a definição de áreas para a execução de pesquisas científicas direcionadas ao manejo diferenciado de cada um destes macrohabitats. Além disso, estes dados são essenciais para compor a base que permite avaliar o papel ecológico da UC e a sua importância para a região.

A EET possui macrohabitats dependentes dos ciclos hidrológicos que ocorrem anualmente em todo o Pantanal. Desse modo, conservar as áreas adjacentes torna-se indispensável para a manutenção deste ecossistema. Os macrohabitats encontrados são uma pequena porção de toda a diversidade de habitats existentes no Pantanal 
mato-grossense, os quais possuem funções ecológicas que necessitam de uma legislação pertinente, de modo a suprir as lacunas para a preservação das áreas úmidas brasileiras. Estudos relacionados aos organismos dependentes ou associados às áreas úmidas são necessários para o desenvolvimento da gestão, do manejo e da conservação da biodiversidade no Pantanal.

\section{AGRADECIMENTOS}

Ao Programa de Pós-Graduação em Ciências Ambientais, da Universidade do Estado de Mato Grosso, pela oportunidade de desenvolver esta pesquisa. À Coordenação de Aperfeiçoamento de Pessoal de Nível Superior, pelo fornecimento de bolsa de pesquisa para AVBF. Ao Instituto Chico Mendes de Conservação da Biodiversidade e aos analistas da Estação Ecológica de Taiamã, SSM Onuma e TDC Pereira, pelo apoio logístico. A BD Vitorino, pelo auxílio nas coletas de campo e na revisão do manuscrito.

\section{REFERÊNCIAS}

AB'SABER, A., 2006. Brasil: paisagens de exceção. O litoral e o Pantanal Mato-Grossense. Patrimônios básicos. Ateliê Editorial, Cotia.

ADAM, E., O. MUTANGA \& D. RUGEGE, 2010. Multispectral and hyperspectral remote sensing for identification and mapping of wetland vegetation: a review. Wetlands Ecology and Management 18(3): 281296. DOI: <https://doi.org/10.1007/s11273-009-9169-z>.

AGÊNCIA NACIONAL DE ÁGUAS (ANA), [s. d.]. Disponível em: <http://www.snirh.gov.br/hidroweb/>. Acesso em: 12 julho 2016.

ARIEIRA, J. \& C. NUNES DA CUNHA, 2012. Estrutura populacional do cambará (Vochysia divergens Pohl, Vochysiaceae), espécie monodominante em floresta inundável no Pantanal Matogrossense. Oecologia Australis 16(4): 819-831. DOI: < http://dx.doi. org/10.4257/oeco.2012.1604.07>.

BLEICH, M. E., R. M. L. SILVEIRA \& F. M. B. NOGUEIRA, 2009. Limnological patterns in northern Pantanal lagoons. Brazilian Archives of Biology and Technology 52(3): 755-764. DOI: <http:// dx.doi.org/10.1590/S1516-89132009000300028> .

BRASIL, 2000. Lei n 9.985, de 18 de julho de 2000. Regulamenta o art. 225, § 1o, incisos I, II, III e VII da Constituição Federal, institui o Sistema Nacional de Unidades de Conservação da Natureza e dá outras providências. Diário Oficial da União, 19 julho 2000. Disponível em: <http://www.planalto.gov.br/ccivil_03/leis/L9985. htm > . Acesso em: 4 abril 2017.
BRASIL/MINISTÉRIO DO MEIO AMBIENTE (MMA), 2015. Recomendação $n^{\circ} 7$ do Comitê Nacional de Zonas Úmidas (CNZU) que dispõe sobre a definição de Áreas Úmidas Brasileiras e sobre o Sistema de Classificação destas áreas. Secretaria de Biodiversidade e Florestas. Disponível em: <http://www.mma. gov.br/areas-protegidas/comit\%C3\%AA-nacional-de-zonas\%C3\%BAmidas\# deliberações> Acesso em: 31 outubro 2017.

BRASIL/MINISTÉRIO DO MEIO AMBIENTE (MMA), 2017. Plano de Manejo da Estação Ecológica de Taiamã: 1-174. Instituto Chico Mendes de Conservação da Biodiversidade (ICMBio/MMA), Brasil.

BRASIL/MINISTÉRIO DO MEIO AMBIENTE(MMA), [s. d.]. Projeto Base Cartográfica Digital Contínua da Amazônia Legal. Disponível em: <http://mapas.mma.gov.br/i3geo/datadownload.htm>. Acesso em: 4 abril 2017.

CALHEIROS, D. F. \& C. J. A. FERREIRA, 1996. Alterações limnológicas no rio Paraguai ("dequada”) e o fenômeno natural de mortandade de peixes no Pantanal Mato-Grossense - MS: 1-51. EMBRAPA-CPAP (Boletim de Pesquisa, 7), Corumbá.

COHEN, J. A., 1960. Coeficient of agreement for nominal scales. Educational and Measurment 20(1): 37-46.

COLGATON, R. G., 1991. A review of assessing the accuracy of classifications of remotely sensed data. Remote Sensing of Environment 49(12): 1671-1678.

CONNELL, J. H. \& M. D. LOWMAN, 1989. Low-density tropical rain forests: some possible mechanism for their existence. The American Naturalist 134: 88-119.

COSTA,M., K.H.TELMER, T.L.EVANS, T.I.R.ALMEIDA\&M.T.DIAKUN, 2015. The lakes of the Pantanal: inventory, distribution, geochemistry, and surrounding landscape. Wetlands Ecology and Management 23(1): 19-39. DOI: <https://doi.org/10.1007/s11273-014-9401-3>.

COWARDIN, I. M., V. CARTER, F. C. GOLET \& E. T. LAROE, 1979. Classification of wetlands and deepwater habitats of the United States: 1-131. US Department of the Interior, Fish and Wildlife Service, Washington.

DA SILVA, C. J., 1984. Nota prévia sobre o significado biológico dos termos usados no Pantanal Mato-grossense, "Batume" e "Diquada". Revista da Universidade Federal de Mato Grosso 4: 30-36.

DA SILVA, C. J. \& F. A. ESTEVES, 1995. Dinâmica das características limnológicas das Baías Porto de Fora e Acurizal (Pantanal de Mato Grosso) em função da variação do nível da água. Oecologia Brasiliensis 1: 47-60.

DA SILVA, C. J. \& J. A. F. SILVA, 1995. No ritmo das águas do Pantanal: 1-210. NUPAUB/USP, São Paulo.

DA SILVA, C. J. \& D. M. FIGUEIREDO, 1999. Variação limnólogica das baías de Chacororé e de Sá Mariana, Pantanal Mato Grossense, Mato Grosso (MT). Revista Mato-Grossense de Geografia 3-4: 57-75.

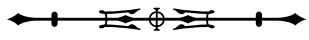


DA SILVA, C. J. \& P. GIRARD, 2004. New challenges in the management of the Brazilian Pantanal and catchment area. Wetlands Ecology and Management 12(6): 553-561. DOI: <https://doi. org/10.1007/s11273-005-1755-0>.

DA SILVA, C. J. \& J. S. SIMONI, 2012. Água, biodiversidade e cultura do Pantanal: estudos ecológicos e etnobiológicos no sistema de Baías Chacororé e Sinhá Mariana: 1. ed.: 1-255. UNEMAT Editora, Cáceres.

FANTIN-CRUZ, I., P. GIRARD, P.ZEILHOFER, W. COLLISCHONN \& C. NUNES DA CUNHA, 2010. Unidades fitofisionômicas em mesoescala no Pantanal Norte e suas relações com a geomorfologia. Biota Neotropica 10(2): 31-38. DOI: <http://dx.doi.org/10.1590/ S1676-06032010000200002>.

FRANCO, M. S. M. \& R. PINHEIRO, 1982. Geomorfologia. In: BRASIL. Projeto RADAMBRASIL: Folha SE 21. Corumbá: 161224. Programa de Integração Nacional (Levantamento de Recursos Naturais, 28), Rio de Janeiro.

GOPAL, B. \& M. SAH, 1995. Inventory and classification of wetlands in India. Vegetatio 118(1-2): 39-48. DOI: < https://doi.org/10.1007/ BF00045189>.

GRIS, D., S. R. V. L. MELO, W. L. C. TABOSA, Y. TAKIGUCHI FILHO, G. A. DAMASCENO JUNIOR, L. B. L. C. COSTA \& M. R. MARQUES, 2016a. Germinação de sementes de Erythrina fusca Lour. (Fabaceae) submetidas à inundação. Anais do Congresso Brasileiro de Áreas Úmidas 3: 1 CD-ROM.

GRIS, D., G. A. DAMASCENO JUNIOR, J. C. CASAGRANDE \& M. R. MARQUES, 2016b. Influência do solo e da inundação sobre a dominância de Erythrina fusca Lour. (Fabaceae) no Pantanal de Cáceres. Anais do Congresso Brasileiro de Áreas Úmidas 3: 1 CD-ROM.

HARRIS, M. B., W. T. TOMAS, G. MOURÃO, C. J. SILVA, E. GUIMARÃES, F. SONODA \& E. FACHIM, 2005. Safeguarding the Pantanal Wetlands: threats and conservation initiatives. Conservation Biology 19(3): 714-720.

HART, T. B., J. A. HART \& P. G. MURPHY, 1989. Monodominant and species-rich forests of the humid tropics: causes for their cooccurrence. The American Naturalist 133(5): 613-633.

IKEDA-CASTRILLON, S. K., C. J. DA SILVA, J. R. C. FERNANDEZ, R. J. NEVES \& I. LEITE, 2011a. Assessment of the arboreal species diversity and correlation between the species distribution and soil characteristics among Paraguay River Islands, section between Caceres and Taiamã Ecological Station, Pantanal, Brazil. Geografia 36: 119-134.

IKEDA-CASTRILLON, S. K., C. J. DA SILVA, J. R. C. FERNANDEZ \& A. K. IKEDA, 2011b. Avaliação da diversidade arbórea das ilhas do rio Paraguai na região de Cáceres, Pantanal Matogrossense, Brasil. Acta Botanica Brasilica 25(3): 672-684. DOI: <http://dx.doi. org/10.1590/S0102-33062011000300021>
INSTITUTO NACIONAL DE METEOROLOGIA (INMET), [s. d.]. Disponível em: <www.inmet.gov.br>. Acesso em: 6 julho 2016.

INSTITUTO NACIONAL DE PESQUISAS ESPACIAIS (INPE), 2006. Tutorial de Geoprocessamento. Disponível em: < http:// www.dpi.inpe.br/spring/portugues/tutorial/index.html > . Acesso em: 4 dezembro 2016.

IRIGARAY, C. T. J. H., 2015. Áreas Úmidas especialmente "des" protegidas no direito brasileiro: o caso do Pantanal Matogrossense e os desafios e perspectivas para sua conservação. Revista de Estudos Sociais 17(34): 203-225.

JUNK, W. J. \& C. J. DA SILVA, 1996. O conceito do Pulso de Inundação e suas implicações para o Pantanal de Mato Grosso. Anais do Simpósio sobre Recursos Naturais e Socioeconômicos do Pantanal: Manejo e Conservação 2: 17-28.

JUNK, W. J., M. T. F. PIEDADE, R. LOURIVAL, F. WITTMANN, P. KANDUS, L. D. LACERDA, R. L. BOZELLI, F. A. ESTEVES, C. NUNES DA CUNHA, L. MALTCHIK, J. SCHÖNGART, Y. SCHAEFFER-NOVELLI \& A. A. AGOSTINHO, 2014. Brazilian wetlands: their definition, delineation, and classification for research, sustainable management, and protection. Aquatic Conservation: Marine and Freshwater Ecosystems 24: 5-22. DOI: <https://doi.org/10.1002/aqc.2386>.

KOPPEN, W., 1948. Climatologia, com um estudio de los clima de la Tierra. FCE, México.

LANDIS, J. R. \& G. G. KOCH, 1977. The measurement of observer agreement for categorical data. Biometrics 33(1): 159-174.

LILLESSAND, T. M. \& R. W. KIEFER, 1999. Remote sensing and image interpretation: 4. ed.: 1-724. John Wiley e Sons, New York.

MENDES, C. L., 1987. Classificação por máxima verossimilhança num ambiente maciçamente paralelo. In: Projeto Estra e Projeto Computação 87/1 786-1. Instituto Nacional de Pesquisas Espaciais, São José dos Campos.

NASCIMENTO, M. T., B. S. S. MARIMON, B. H. MARIMONJUNIOR, C. NUNES DA CUNHA \& D. M. VILLELA, 2015 Florestas monodominantes no Brasil: estudos de caso. In: P. V. EISENLOHR, J. M. FELFILI, M. M. R. F. MELO, L. A. ANDRADE \&J. A. A. MEIRA NETO (Ed.): Fitossociologia no Brasil: métodos e estudos de casos: v. 2: 1-566. Editora UFV, Viçosa.

NAVARRO, G. \& M. MALDONADO, 2002. Geografía ecológica de Bolivia: vegetación y ambientes acuáticos: 1-719. Centro de Ecología Simón I, Cotchabamba, Bolivia.

NEIFF, J. J., 2001. Humedales de la Argentina: sinopsis, problemas y perspectivas futuras. In: A. F. CIRELLI (Ed.): El agua en Iberoamérica. Funciones de los humedales, calidad de vida y agua segura: 83-112. CYTED, Buenos Aires.

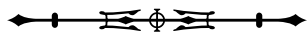


NEVES, S. M. A. S., C. B. M. CRUZ, R. J. NEVES \& A. SILVA, 2009. Geotecnologias aplicadas na identificação e classificação das unidades ambientais do Pantanal de Cáceres/MT - Brasil. Geografia 34: 795-805.

NUNES DA CUNHA, C., P. RAWIEL, K. M. WANTZEN, W. J. JUNK \& A. LEMES DO PRADO, 2006. Mapping and characterization of vegetation units by means of Landsat imagery and management recommendations for the Pantanal of Mato Grosso (Brazil), North of Poconé. Amazoniana 19(1-2): 1-32.

NUNES DA CUNHA, C. \&W. J. JUNK, 2009. Landscape units of the Pantanal: structure, function, and human use. In: W. J. JUNK, C. J. DA SILVA, C. NUNES DA CUNHA \& K. M. WANTZEN (Ed.): The Pantanal: ecology, biodiversity and sustainable management of a large neotropical seasonal wetland: 127-141. Pensoft Publishers, Sofia.

NUNES DA CUNHA, C. \& W. J. JUNK, 2014. A classificação dos macrohabitats do Pantanal Mato-grossensse. In: C. NUNES DA CUNHA, M. T. F. PIEDADE \& W. J. JUNK (Ed.): Classificação e delineamento das áreas úmidas brasileiras e de seus macrohabitas: 1-156. EdUFMT, Cuiabá.

NUNES DA CUNHA, C., M. T. F. PIEDADE \& W. J. JUNK, 2014. Classificação e delineamento das áreas úmidas brasileiras e de seus macrohabitas: 1-156. EdUFMT, Cuiabá.

OLLIS, D. J., J. L. EWART-SMITH, J. A. DAY, N. M. JOB, D. M. MACFARLANE, C. D. SNADDON, E. J. J. SIEBEN, J. A. DINI \& N. MBONA, 2015. The development of a classification system for inland aquatic ecosystems in South Africa. Water SA 41(5): 727-745. DOI: <http://dx.doi.org/10.4314/wsa.v41i5.16>.

OZESMI, S. L. \& M. E. BAUER, 2002. Satellite remote sensing of wetlands. Wetlands Ecology and Management 10(5): 381-402. DOI: <https://doi.org/10.1023/A:1020908432489>.

PAIVA, S. L. P., S. M. A. S. NEVES, C. C. MUNIZ, R. J. NEVES \& A. O. FURLAN, 2014. Uso da terra e cobertura vegetal nas unidades geomorfológicas do corredor fluvial do Rio Paraguai no município de Cáceres/MT, Brasil. Revista Geonorte 10(1): 687-692.

PEREIRA, G., E. S. CHÁVEZ \& M. E. S. SILVA, 2012. O estudo das unidades de paisagem do bioma Pantanal. Revista Ambiente \& Água 7(1): 89-103. DOI: <http://dx.doi.org/10.4136/ambiagua.826>.

PINTO, A. A., C. J. DA SILVA, P. GIRARD, M. SOUZA \& F. NOGUEIRA, 1999. The flood pulse and the limnological of the Sinhá Mariana baía in the Mato Grosso pantanal, Brazil. Revista Boliviana de Ecologia y Conservacion Ambiental 6: 19-26.

PIVARI, M. O., V. J. POTT \& A. POTT, 2008. Macrófitas aquáticas de ilhas flutuantes (baceiros) nas sub-regiões do Abobral e Miranda, Pantanal, MS, Brasil. Acta Botanica Brasilica 22(2). DOI: <http:// dx.doi.org/10.1590/S0102-33062008000200023>.
PIZZATO, J. A., R. DALLACORT, R. C. TIEPPO, A. J. MODOLO, C. CREMON \& P. S. P. MOREIRA, 2012. Distribuição e probabilidade de ocorrência de precipitação em Cáceres (MT). Pesquisa Agropecuária Tropical 42(2): 137-142.

PONZONI, F. J. \& P. HERNANDEZ-FILHO, 1989. A fisionomia da cobertura vegetal do Parque Nacional do Pantanal Mato-grossense (PNPM) identificada através do sensor TM/Landsat: uma análise multitemporal. Anais do Simpósio Brasileiro de Sensoriamento Remoto 5: 670-674.

POTT, V. J. \& A. POTT, 2000. Plantas aquáticas do Pantanal: 1. ed. EMBRAPA, Corumbá.

POTT, A., C. NUNES DA CUNHA, V. J. POTT, E. A. SILVEIRA \& A. L. B. SARTORI, 2001. Avaliação ecológica rápida: componente botânica - Parque Nacional do Pantanal mato-grossense e entorno. In: BRASIL. Plano de Manejo do Parque Nacional do Pantanal Mato-grossense: anexo 4. MMA, Brasília.

POTT, V. J., A. POTT, L. C. P. LIMA, S. N. MOREIRA \& A. K. M. OLIVEIRA, 2011. Aquatic macrophyte diversity of the Pantanal wetland and upper basin. Brazilian Journal Biology 71(1): 255-263.

RAVAGLIA, A. G., S. A. SANTOS, B. M. A. SORIANO, O. DANIEL, L. C. FREITAS, L. A. PELLEGRIN, W. M. TOMAS, L. G. RODELA, A. A. BUENOSOBRINHO \& M. T. B. D. ARAÚJO, 2011. Mapeamento das unidades de paisagem das sub-regiões da Nhecolândia e Poconé, Pantanal Mato-grossense: 1-16. EMBRAPA PANTANAL (Boletim de Pesquisa e Desenvolvimento, 105), Corumbá.

REBELLATO, L. \& C. NUNES DA CUNHA, 2005. Efeito do "fluxo sazonal mínimo da inundação" sobre a composição e estrutura de um campo inundável no Pantanal de Poconé, MT, Brasil. Acta Botanica Brasilica 19(4): 789-799. DOI: <http://dx.doi.org/10.1590/S010233062005000400015>

REBELLATO, L., C. NUNES DA CUNHA \& J. E. C. FIGUEIRA, 2012. Respostas da comunidade herbácea ao pulso de inundação no Pantanal de Poconé, Mato Grosso. Oecologia Australis 16(4): 797-818.

ROSA, R., 2005. Geotecnologias na geografia aplicada. Revista do Departamento de Geografia 16: 81-90.

ROSA, R., 2009. Introdução ao sensoriamento remoto: 7. ed. EDUFU, Uberlândia.

SANTOS, J. S., G. PEREIRA, Y. E. SHIMABUKURO \& B. F. T. RUDORFF, 2009. Identificação de áreas alagadas no Bioma Pantanal - Brasil - utilizando dados multitemporais Terra/Modis. Geografia 34: 745-755.

SANTOS, S. A., L. G. RODELA, W. TOMAS, C. NUNES DA CUNHA, A. RAVAGLIA \& L. A. PELLEGRIN, 2009. Mapeamento das unidades de paisagens, fitofisionomias e forrageiras chaves da sub-região de Poconé, Pantanal, MT. Anais do Simpósio de Geotecnologias no Pantanal 2: 613-622.

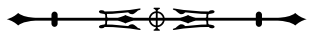


SANTOS, L., C. A. G. P. ZAMPARONI \&J. C. O. SOARES, 2012. O ritmo pluviométrico na região de Cáceres-MT no período compreendido entre a série histórica de 1971 a 2010. Revista Geonorte 1(5): 1091-1102.

SCOTT, D. A. \& T. A. JONES, 1995. Classification and inventory of wetlands: a global overview. Vegetatio 118(1-2): 3-16. DOI: $<$ https://doi.org/10.1007/BF00045186>.

SILVA, J. S. V. \& M. M. ABDON, 1998. Delimitação do Pantanal brasileiro e suas sub-regiões. Pesquisa Agropecuária Brasileira 33: 1703-1711.

SILVA, J. S. V., M. M. ABDON, A. M. SILVA \& L. S. CUNHA, 2006. Estado da arte do mapeamento da vegetação no Pantanal brasileiro. Anais do Seminário de Atualização em Sensoriamento Remoto e Sistemas de Informações Geográficas Aplicados à Engenharia Florestal 7: 65-72.

SILVA, A., M. L. ASSINE, E. E. SOUZA FILHO, S. B. CUNHA \&H. ZANI, 2006. Compartimentação geomorfológica do rio Paraguai na borda norte do Pantanal, município de Cáceres, MT. Anais do Simpósio de Geotecnologias no Pantanal 1: 257-264.

SiLVA, M. P., R. MAURO, G. MOURÃO \& M. COUTINHO, 2000. Distribuição e quantificação de classes de vegetação do Pantanal através de levantamento aéreo. Revista Brasileira de Botânica 23(2): 143-152. DOI: <http://dx.doi.org/10.1590/S010084042000000200004>.
SOUZA, C. A. \& S. B. CUNHA, 2007. Pantanal de Cáceres - MT: dinâmica das margens do rio Paraguai. Seção Três Lagoas. Revista Eletrônica da Associação dos Geógrafos Brasileiros 4(5): 18-41.

SOUZA, C. A., G. R. S. LEANDRO \& J. B. SOUSA, 2014. Migração lateral do canal do rio Paraguai entre a cidade de Cáceres e a Estação Ecológica de Taiamã - Mato Grosso. GEOgraphia 16(31): 123-141.

UNITED STATES GEOLOGICAL SURVEY (USGS), [s. d.]. Earth Explorer. Disponível em: <http://earthexplorer.usgs.gov/>. Acesso em: 10 julho 2016.

VELOSO, H. P., A. L. T. RANGEL-FILHO \& J. C. A. LIMA, 1991. Classificação da vegetação brasileira adaptada a um sistema universal. IBGE, Rio de Janeiro.

WANTZEN, K. M., E. DRAGO \& C. J. DA SILVA, 2005. Aquatic habitats of the Upper Paraguay River-Floodplain-System and parts of the Pantanal. Ecohydrology \& Hydrobiology 5(2): 107-126.

WARNER, B. G. \& C. D. A. RUBEC, 1997. The Canadian Wetland Classification System: 2. ed. Wetlands Research Centre, University of Waterloo, Waterloo, Ontario.

WIENS, J. A., N. C. STENSETH, B. V. HORNE \& R. A. IMS, 1993. Ecological mechanisms and landscape ecology. Oikos 66(3): 369-380. 